\title{
ORIGINAL ARTICLE Spinal cord injury without radiographic abnormality (SCIWORA) in adults: MRI type predicts early neurologic outcome
}

\author{
CK Boese ${ }^{1}$, D Müller ${ }^{2}$, R Bröer ${ }^{1}$, P Eysel ${ }^{1}$, B Krischek ${ }^{3}$, HC Lehmann ${ }^{4}$ and P Lechler ${ }^{5}$
}

Objectives: The present study aimed to analyse the clinical and neuroimaging features of a consecutive series of adult patients with spinal cord injury without radiographic abnormality (SCIWORA) receiving early magnetic resonance imaging (MRI), and to apply the recently proposed MRI classification system.

Methods: Grade of neurologic impairment at admission and discharge was reported according to the American Spinal Injury Association Impairment Scale (AIS). A detailed analysis and categorisation of the extra- and intramedullary MRI findings was performed, and the relationship between imaging type and neurological outcome was described.

Results: Twenty-six adult patients ( 17 male and 9 female) with SCIWORA were identified (mean age of 52 years). The distribution of the initial AIS grade was $8 \%$ A $(n=2), 19 \%$ B $(n=5), 31 \% \mathrm{C}(n=8)$ and $42 \% \mathrm{D}(n=11)$ at admission and $15 \%(n=4) \mathrm{C}, 58 \%$ $(n=15) \mathrm{D}$ and $27 \%(n=7) \mathrm{E}$ at discharge, respectively. Type I SCIWORA was found in $23 \%(n=6)$ and type II in $77 \%(n=20)$ (Ila: 0\%, Ilb: 25\%, Ilc: 75\%). The mean improvement of AIS grade in patients with type I lesions was 1.5 (median 1, range 1-3) and 0.9 (median 1 , range $0-3$ ) in type II.

Conclusion: The findings underline the prognostic role of early MRI for adult patients with SCIWORA and support the use of the recently introduced MRI classification system.

Level of evidence: Prognostic study, level III.

Spinal Cord (2016) 54, 878-883; doi:10.1038/sc.2016.13; published online 16 February 2016

\section{INTRODUCTION}

Traumatic spinal cord injury (SCI) describes neurological impairment caused by blunt or penetrating trauma to the spinal cord and the surrounding soft and osseous tissues. In majority of the patients with SCI, lesions to the spinal column (that is, fractures and dislocations) are detected by conventional radiographs or computed tomography (CT). However, a subpopulation of patients presents with a clinico-radiological mismatch, previously termed spinal cord injury without radiological abnormality (SCIWORA). ${ }^{1}$ Although the introduction of magnetic resonance imaging (MRI) allowed the visualisation of spinal soft-tissue lesions and intramedullary abnormalities, there remained a significant fraction of patients presenting with a discrepancy between neurological impairment and detectable MRI findings. ${ }^{2-4}$ Furthermore, the prognostic value of extra- and intramedullary MRI findings in traumatic SCI presents an ongoing matter of debate. ${ }^{3,5}$ To further improve the interpretability and comparability of MRI findings in patients with a clinicoradiological mismatch, an MRI-based classification system has been introduced. ${ }^{4,6}$

The present study aimed (1) to further characterise the clinical features of adult patients presenting with SCIWORA, (2) to apply the MRI classifications system in a consecutive cohort and (3) to assess its prognostic value in the prediction of the early neurological outcome in this population.

\section{PATIENTS AND METHODS}

For the present study, SCIWORA was defined as traumatic SCI with neurological impairment at admission to the hospital and exclusion of major fractures or dislocations to the spine; minor osseous lesions without a relation to the neurological affected region were not excluded (that is, fracture of a transverse process).

The hospital information system (HIS; ORBIS, Agfa Healthcare GmbH, Bonn, Germany) of a university hospital in central Europe was retrospectively searched for patients with traumatic spinal cord injuries. The HIS was implemented in 2005, and the search included the period from 1 January 2005 to 1 July 2014. Identification was based on the 10th edition of the International Statistical Classification of Diseases and Related Health Problems German modification (ICD-10). The codes S14. ${ }^{*}, \mathrm{~S} 24 .^{*}, \mathrm{~S} 34 .^{*}$ and T09.3 were searched. Inclusion criteria were adult patient at admission (age $\geqslant 17$ years) with mature skeleton, traumatic SCI, radiograph and/or CT and MRI of the spine. Exclusion criteria were age $<17$ years or immature skeleton, non-traumatic SCI, non-existent or missing radiograph and/or CT and/or MRI of the spine, incomplete clinical data, fractures or dislocations of the spine (Figure 1).

For all included patients, the following data were extracted: case number, patient ID, name, sex, date of birth, age at admission, date of admission and discharge, trauma mechanism, length of inpatient stay, duration of ventilation,

${ }^{1}$ Department of Orthopaedic and Trauma Surgery, University Hospital of Cologne, Cologne, Germany; ${ }^{2}$ Department of Radiology, University of Cologne, Cologne, Germany; ${ }^{3}$ Department of Neurosurgery, University of Cologne, Cologne, Germany; ${ }^{4}$ Department of Neurology, University Hospital of Cologne, Cologne, Germany and ${ }^{5}$ Center for Orthopaedics and Trauma Surgery, University of Giessen and Marburg, Marburg, Germany

Correspondence: Dr CK Boese, Department of Orthopaedic and Trauma Surgery, University Hospital of Cologne, Joseph-Stelzmann-Strasse 9, Cologne 50924, Germany.

E-mail: christoph.boese@uk-koeln.de

Received 17 September 2015; revised 15 December 2015; accepted 18 December 2015; published online 16 February 2016 


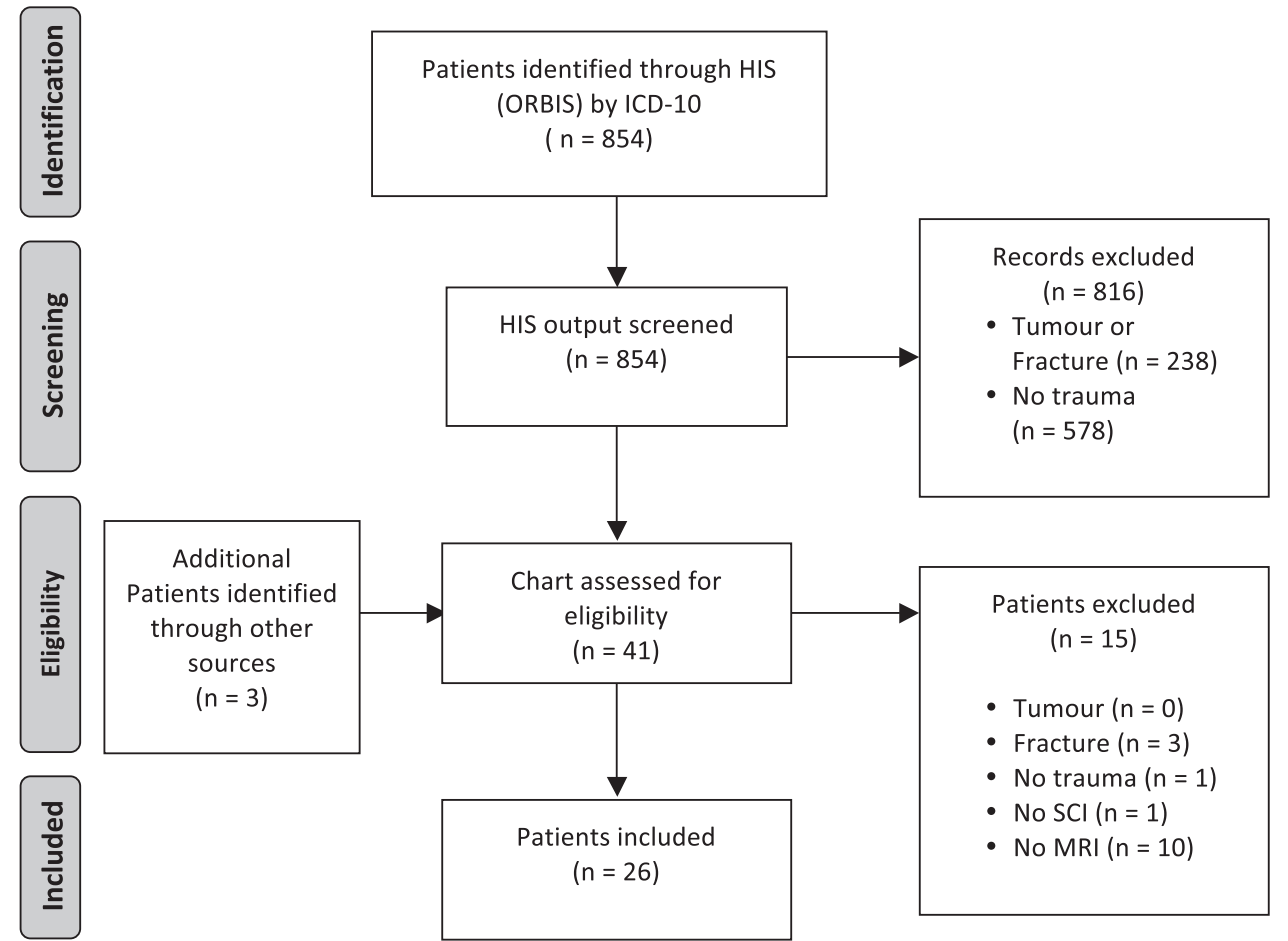

Figure 1 Flow chart depicting the inclusion and exclusion process.

Table 1 Classification of MRI types in adult patients with SCIWORA

\begin{tabular}{ll}
\hline MRI type & MRI finding \\
\hline Type I & No detectable abnormalities \\
Type Ila & Intraneural abnormalities \\
Type IIb & Extraneural abnormalities \\
Type Ilc & Extraneural and intraneural abnormalities
\end{tabular}

Abbreviations: MRI, magnetic resonance imaging; SCIWORA, spinal cord injury without radiological abnormality.

Adapted from Boese and Lechler. ${ }^{4}$

therapeutic interventions, American Spinal Injury Association Impairment Scale (AIS) at admission and discharge, modified Frankel score at admission and discharge, time to MRI (in days) and available outpatient follow-up data.

Patients were treated with physiotherapy during their hospital stay on a daily basis depending on their individual needs. Those with inability to walk at discharge were transferred to neuro-rehabilitation facilities.

\section{MRI and classification of MRI patterns}

In 21 patients, MRI scans were stored in a picture-archiving and communication system (IMPAX, AGFA HealthCare GmbH, Bonn, Germany). In five patients, MRI data were available as print. MRI scans were analysed following a standardised protocol. Intramedullary findings were classified as either oedema or haemorrhage in sagittal T1- and T2-weighted MRI images: oedema was defined as hyperintense T2 signals (central and peripheral) and normal T1 signal; haemorrhage was defined as inhomogenous T1 and hypointense (central) T2 signals with a rim of hyperintense rim. ${ }^{7}$ Extramedullary findings were classified as stenosis, intraspinal and prevertebral haematoma, injury to the spinal ligaments and minor fractures. Finally, the MRI type was classified according to Boese and Lechler (Table 1). ${ }^{4}$ Sagittal T1-weighted, T2-weighted and Short Tau Inversion Recovery sequences were available for analysis in all patients. Images were acquired using five MRI machines (1.5 Tesla Philips Medical Systems, Philips GmbH Market DACH Healthcare, Hamburg, Germany: Intera $(n=19)$, Ingenia $(n=2)$ and Achieva $(n=4) ; 1$ Tesla Philips Medical Systems Panorama HFO, Philips GmbH Market DACH Healthcare $(n=1)$ ).

\section{Statistics}

Statistical analysis was performed using IBM SPSS Statistics 22 (IBM Corporation, Armonk, NY, USA). Descriptive analysis included the presentation of absolute mean values and ranges of the measured variables. AIS grades were transformed into numbers $(\mathrm{A}=1, \mathrm{~B}=2$, and so on), means and medians and ranges were given.

\section{Ethics}

The study protocol followed the Declaration of Helsinki and was approved by the local ethics committee (14-162). Because of the retrospective study design, no informed consent was necessary.

\section{RESULTS}

Demographic and clinical characteristics of adult patients with SCIWORA

A total of 26 (17 male and 9 female) adult patients with SCIWORA with a mean age of 52 years (range: 17-80) and a mean inpatient stay of 11.3 days (range: 2-37) were included. Type I SCIWORA had a mean length of hospital stay of 3.3 days (range: $2-6$; s.d. 2.1) compared with type IIb and IIc with means of 12 days (range: 2-18; s.d. 6.6) and 14.8 days (range: 3-37; s.d. 10.1), respectively.

The detailed demographic and clinical characteristics of the cohort are shown in Table 2. The mean delay to spinal MRI imaging was 1.2 days (range: $0-11$ days); twenty patients received MRI within $24 \mathrm{~h}$, one within $48 \mathrm{~h}$, three within 3 days, one within 5 days and one patient 11 days following the trauma. CT before MRI was performed in 21 patients. Nine patients were monitored on ICU, and four patients needed mechanical ventilation for a mean time of 7.5 days (range: 1-21). Eighteen patients received intravenous corticosteroids and eight patients were treated surgically. In seven patients, a cervical orthesis was employed. 


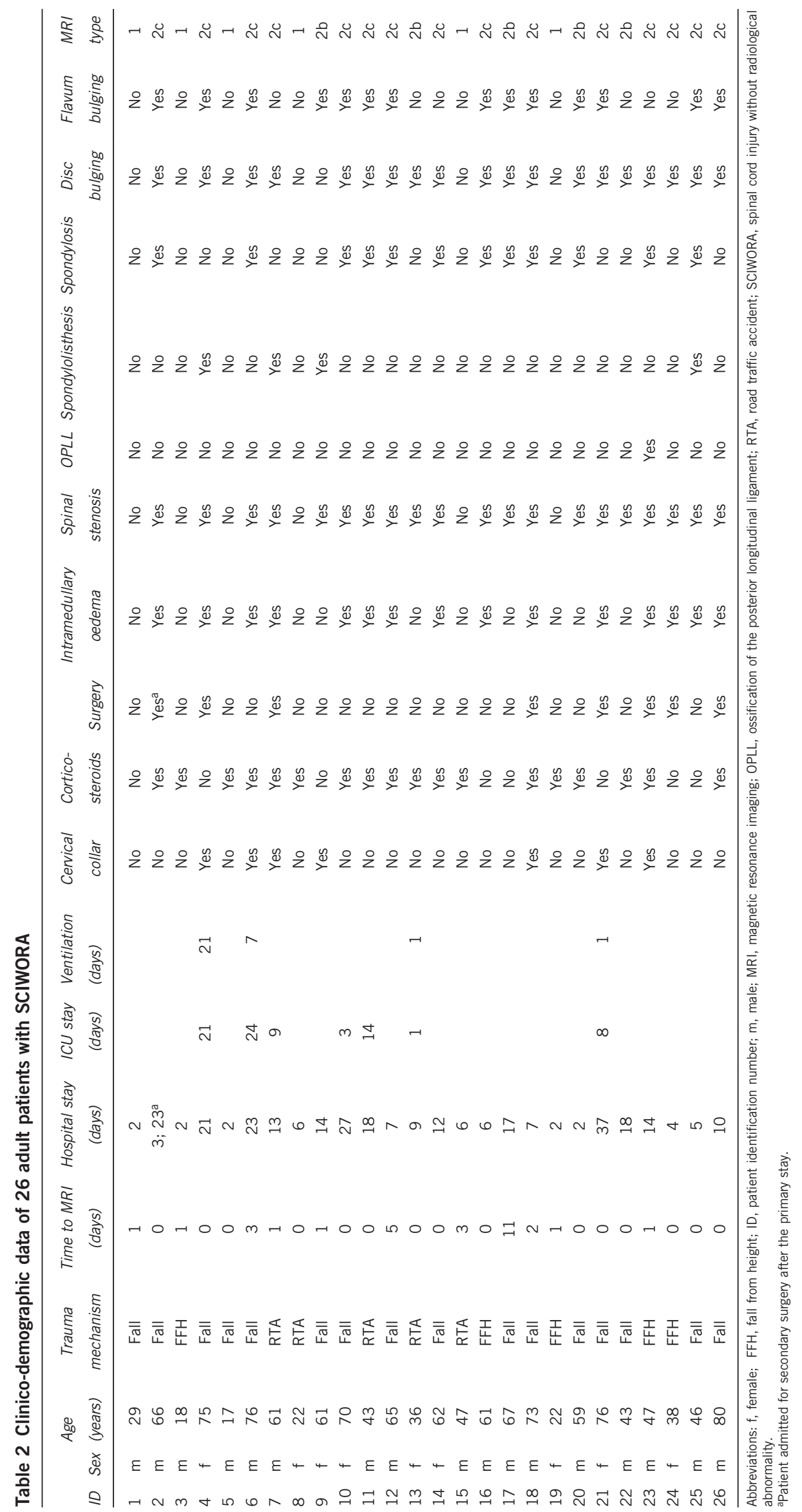




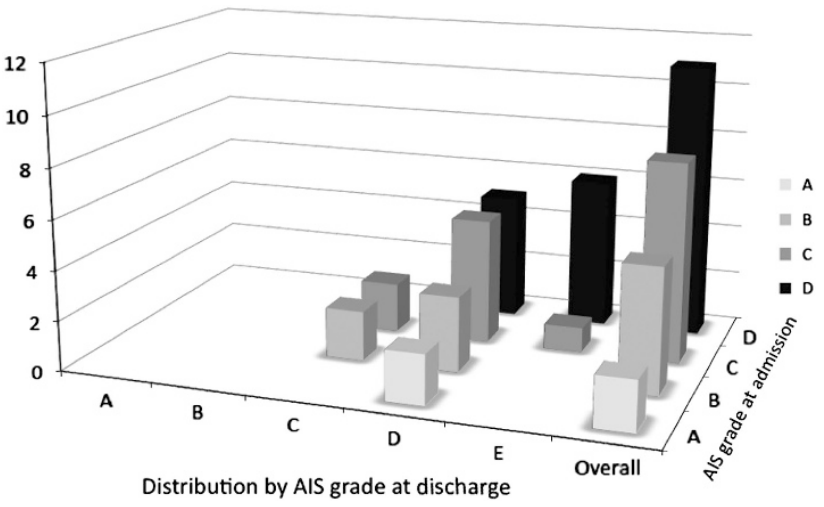

Figure 2 Neurologic impairment at admission and at discharge of adult patients with SCIWORA assessed by AIS. Distribution of AIS at admission ( $z$ axis) relative to distribution of AIS at discharge ( $x$ axis). ${ }^{4}$

\section{Neurologic impairment}

All included patients presented with a cervical SCI. Neurologic impairment at admission and at discharge is shown in Figure 2 and Table 3.

\section{MRI findings and classification of imaging type}

The detailed analysis of the observed MRI findings is given in Table 4. In three patients, five minor fractures not related to the SCI were observed (one spinal process fracture, one minimal anterior column impression and three transverse process fractures). Fifty-eight percent $(n=15)$ patients presented with intramedullary oedema, whereas no bleeding, contusion or transection was found. Patients were grouped into MRI types according to the following: ${ }^{4}$ type I was found in $23 \%$ $(n=6)$ and type II in $77 \%(n=20)$ (IIa: $0 \%$, IIb $25 \%$, IIc $75 \%)$.

\section{Relation between MRI type and neurologic impairment}

The relation between AIS and imaging type is shown in Figures 3a-d. The mean improvement of AIS grade in patients with type I lesions was 1.5 (median 1 , range $1-3$ ) and 0.9 (median 1 , range $0-3$ ) in type II. Although type IIb improved by 1.4 (median 2, range 0-2), type IIc was associated with a mean increase of 0.7 AIS grades (median 1, range $0-3$ ). All eight patients who received surgical decompression presented with SCIWORA type IIc.

\section{DISCUSSION}

The diagnostic and therapeutic approach to adult patients with SCI presenting with a clinico-radiological mismatch remains to be an ongoing challenge to our field. ${ }^{4}$ Although early MRI has been shown to influence treatment decisions in patients with SCI without major fractures or dislocations, ${ }^{8}$ there remains a paucity of knowledge regarding its role in the prognostication of the neurological outcome. To further improve the reliability and comparability of MRI findings in patients with SCIWORA, a classification system has been introduced recently. ${ }^{4,6}$ In contrast to the previously reported grading systems limited to either extramedullary ${ }^{9}$ or intramedullary MRI abnormalities, ${ }^{10}$ the recent classification system accounts for both locations and for cases without detectable MRI findings. ${ }^{4}$ Here, imaging type I was defined as a normal MRI scan, whereas type II comprises three pathological subtypes (see Table 1). Although this classification system has been successfully used to analyse pooled data from publications reporting on paediatric and adult SCIWORA, ${ }^{4,6}$ its prognostic value and real world applicability have not been tested yet.
Table 3 Individual courses of 26 adult patients with SCIWORA including AIS grade and the modified Frankel scale

\begin{tabular}{|c|c|c|c|c|}
\hline \multirow[t]{2}{*}{ ID } & \multicolumn{2}{|c|}{ Neurolog. status at admission } & \multicolumn{2}{|c|}{ Neurolog. status at discharge } \\
\hline & AIS grade & Modified Frankel Scale & AIS grade & Modified Frankel Scale \\
\hline 1 & C & C & $E$ & $E$ \\
\hline 2 & C & C & D & D1 \\
\hline 3 & C & C & $\mathrm{D}$ & D2 \\
\hline 4 & C & C & C & C \\
\hline 5 & $\mathrm{D}$ & D3 & $E$ & $E$ \\
\hline 6 & $A$ & $A$ & $\mathrm{D}$ & D1 \\
\hline 7 & C & C & C & C \\
\hline 8 & A & A & $\mathrm{D}$ & D2 \\
\hline 9 & D & D1 & $\mathrm{D}$ & D2 \\
\hline 10 & C & C & $\mathrm{D}$ & D2 \\
\hline 11 & B & B & C & C \\
\hline 12 & $D$ & D2 & $D$ & D2 \\
\hline 13 & B & B & $D$ & D1 \\
\hline 14 & $D$ & D1 & $\mathrm{D}$ & D3 \\
\hline 15 & D & D2 & $E$ & $E$ \\
\hline 16 & $\mathrm{D}$ & D2 & $E$ & $E$ \\
\hline 17 & B & $B$ & $\mathrm{D}$ & D2 \\
\hline 18 & $\mathrm{D}$ & NA & $\mathrm{D}$ & NA \\
\hline 19 & $\mathrm{D}$ & D3 & $E$ & $E$ \\
\hline 20 & $D$ & D3 & $E$ & $E$ \\
\hline 21 & C & C & $\mathrm{D}$ & D2 \\
\hline 22 & B & $B$ & $D$ & D2 \\
\hline 23 & $D$ & D2 & $E$ & $E$ \\
\hline 24 & $\mathrm{D}$ & D1 & $\mathrm{D}$ & D2 \\
\hline 25 & C & $C$ & $D$ & NA \\
\hline 26 & B & B & $C$ & C \\
\hline
\end{tabular}

Abbreviations: AIS, American Spinal Injury Association Impairment Scale; NA, not available; SCIWORA, spinal cord injury without radiological abnormality.

Although a correlation between neurological outcome and MRI pattern has been described previously, ${ }^{4,5,11-18}$ this finding could not be confirmed by others. ${ }^{2}$ The present study reports the existence of a relation between imaging type and neurological outcome in adult patients with SCIWORA. Furthermore, our data support the previously reported superior prognosis of patients presenting with SCIWORA type I (no detectable neuroimaging abnormalities) over patients presenting with type II lesions. ${ }^{4}$ The subsequent analysis of the type II subgroup revealed a higher risk of persistent neurological impairment for patients with combined extra- and intraneural abnormalities (type IIc) compared with those with isolated extraneural abnormalities (type IIb).

In contrast to children with SCIWORA, degenerative changes of the spine and subsequent stenosis to the spinal channel are frequently found in adults presenting with a clinico-radiological mismatch. ${ }^{19}$ Importantly, pre-existing spinal stenosis has been proposed to be a risk factor for the development of $\mathrm{SCI}^{9,20,21}$ and SCIWORA. ${ }^{4}$ In the analysed population, the detection of extramedullary abnormalities (type II) was associated with an inferior outcome when compared with patients without extramedullary abnormalities. Despite the fact that all surgical interventions were performed in patients with type IIc lesions, this imaging type was associated with a high risk of poor neurological outcome.

Kulkarni et al. ${ }^{7}$ described three imaging patterns of intramedullary abnormalities (cord haemorrhage, oedema and mixed type), and their prognostic value has been the focus of a number of reports. ${ }^{8,22}$ In the 
Table 4 Surgical therapy in patients with SCIWORA

\begin{tabular}{lccl}
\hline ID & Sex & MRI type & Operation \\
\hline 2 & $\mathrm{~m}$ & $2 \mathrm{c}$ & Corporectomy and ventral stabilisation with plate and autologous bone graft \\
4 & $\mathrm{f}$ & $2 \mathrm{c}$ & Corporectomy and ventral stabilisation with plate and autologous bone graft \\
7 & $\mathrm{~m}$ & $2 \mathrm{c}$ & Corporectomy and ventral stabilisation with plate and autologous bone graft and cage implantation \\
18 & $\mathrm{~m}$ & $2 \mathrm{c}$ & Corporectomy and ventral stabilisation with plate and cage implantation \\
21 & $\mathrm{f}$ & $2 \mathrm{c}$ & Primary: dorsal decompression; secondary ventral plate fixation \\
23 & $\mathrm{~m}$ & $2 \mathrm{c}$ & Corporectomy and ventral stabilisation with plate and cage implantation \\
24 & $\mathrm{f}$ & $2 \mathrm{c}$ & Ventral discetomy, decompression and cage implantation with ventral plate fixation \\
\hline
\end{tabular}

Abbreviations: MRI, magnetic resonance imaging; SCIWORA, spinal cord injury without radiological abnormality.

a

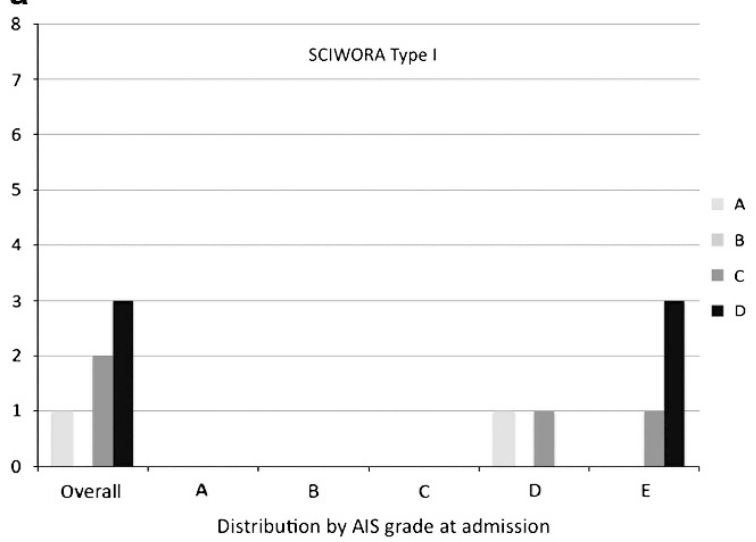

C

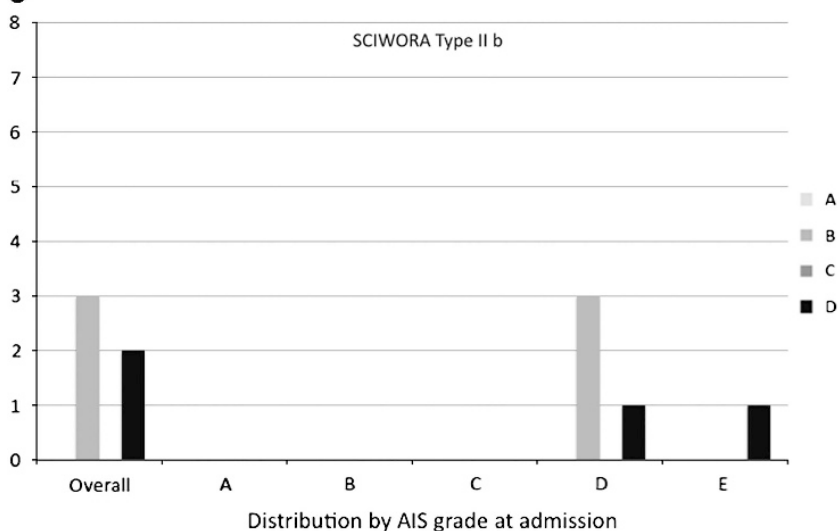

b
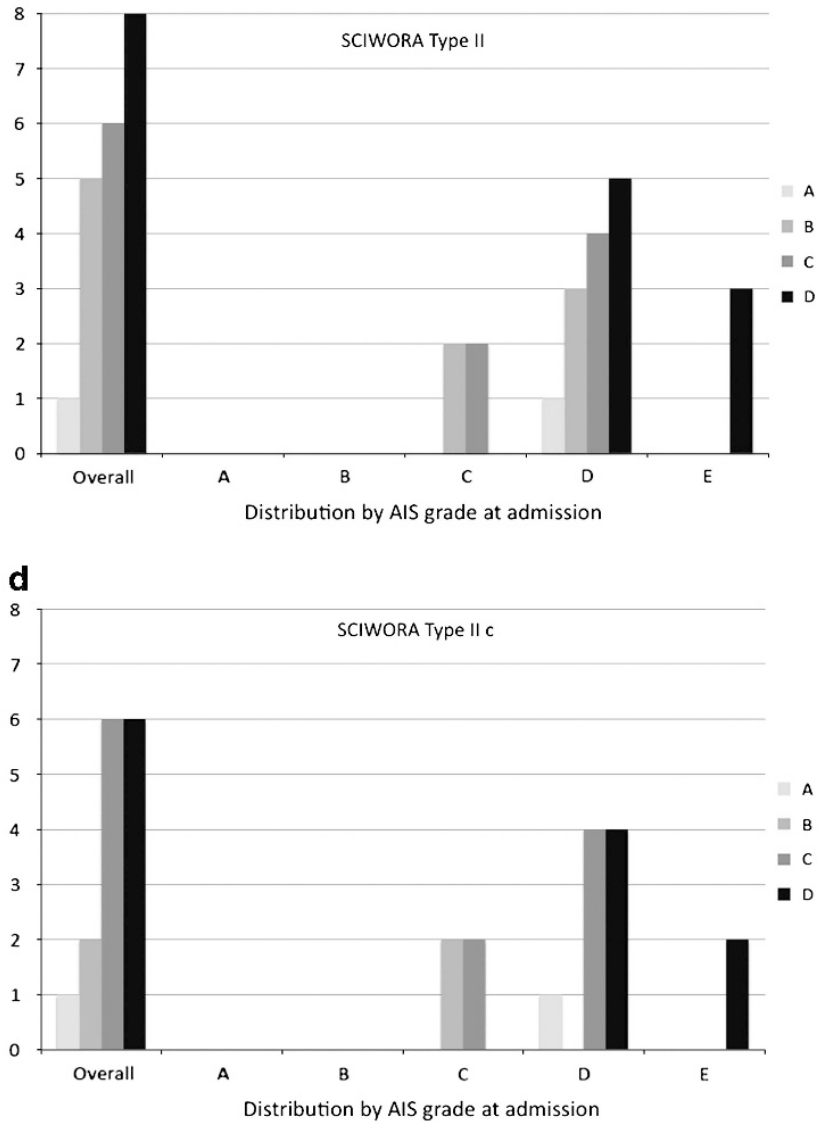

Figure 3 (a-d) Distribution of AIS at admission and at discharge final relative to MRI type. ${ }^{4}$ (a) Type I. (b) Type II. (c) Type IIb. (d) MRI IIc.

present study, the detection of intramedullary abnormalities was limited to oedemas, whereas no haematomas could be found. The presence of an intramedullary oedema in combination with an extramedullary finding was associated with a poorer neurologic outcome compared with patients presenting with an isolated extramedullary finding and those without any detectable neuroimaging abnormalities.

The treatment in SCIWORA is still based on individual decisions and includes conservative and operative options. In our cohort, surgical procedures were performed in type IIc SCIWORA only. No case of type I or type IIb received surgery. The surgery was indicated on an individual basis, and only patients with spinal channel stenosis and myelopathy signals on MRI were operated on. The types of surgery were ventral approaches to the cervical spine in 7/7 cases (one operated on during a secondary hospital stay). One patient received dorsal decompression with laminectomy in the first operation and secondary ventral plate fixation; this patient had the longest hospital stay of 37 days including 8 days in the ICU. Interestingly, the mean age of surgical patients was 62.3 years (range 38-76) contrasting 46.9 years (range 17-76) in the conservative group. The use of corticosteroids was based on individual decisions of the treating surgeons and neurosurgeons. Seventeen patients received varying doses of corticosteroids. Of these, three underwent surgery. The application of cervical collars was inhomogeneous. Five of seven surgically treated patients received collars for the post-operative phase, whereas only two patients with a conservative approach were treated with cervical collars. 
The authors are aware of the limitations of this report:

First, our findings are based on a retrospective study design and should be confirmed by future prospective observational investigations. However, because of the overall low incidence rate of adult SCIWORA, we recommend a prospective multicentre study of cases presenting with a clinico-radiological mismatch following SCI.

Because of the lack of a consistent rationale determining the choice between surgical or non-surgical treatment and the inhomogeneous application of corticosteroids, our results cannot provide guidance on the therapeutic management of adult patients with SCIWORA.

Furthermore, our study does not include long-term follow-up data, thus limiting the interpretability of the presented outcome data.

Importantly, the study was performed in central Europe, thus limiting the transferability of our findings to populations with different ethnic backgrounds. Consequently, the imaging features of the reported cohort are in contrast to recent reports on Asian adult patients with SCIWORA, with high prevalence rates of ossification of the posterior longitudinal ligament. ${ }^{23}$

Next, although high-field MRI scanners and specialised sequences might provide additional information on intra- and extramedullary pathologies in patients with SCIWORA, ${ }^{24}$ this study was restricted on widely available MRI technology and standard sequences.

The major strength of the present study is its large number of the included adult patients with SCIWORA, as well as the thorough analysis and detailed presentation of the neurologic and imaging characteristics resulting in an excellent reproducibility and interpretability of our data.

Finally, the application of the classification system allowed the reliable assessment of the correlation between neurologic impairment and imaging abnormalities in adult patients with SCIWORA.

\section{CONCLUSION}

The findings of our study underline the prognostic role of early MRI for adult patients with SCIWORA and support the application of the recently introduced MRI classification system.

\section{DATA ARCHIVING}

There were no data to deposit.

\section{CONFLICT OF INTEREST}

The authors declare no conflict of interest.

\section{AUTHOR CONTRIBUTIONS}

$\mathrm{CKB}$ and $\mathrm{RB}$ performed data collection, $\mathrm{CKB}, \mathrm{RB}$ and $\mathrm{PL}$ contributed to data analysis, $\mathrm{CKB}$ and PL wrote the manuscript, JD, RB, PE, BK, HCL and PL interpreted the data and critically reviewed the manuscript, all authors read and approved the final version of the manuscript.
1 Pang D, Pollack IF. Spinal cord injury without radiographic abnormality in children-the SCIWORA syndrome. J Trauma 1989; 29: 654-664.

2 Liu Q, Liu Q, Zhao J, Yu H, Ma X, Wang L. Early MRI finding in adult spinal cord injury without radiologic abnormalities does not correlate with the neurological outcome: a retrospective study. Spinal Cord 2015; 53: 750-753.

3 Boese CK, Nerlich M, Klein SM, Wirries A, Ruchholtz S, Lechler P. Early magnetic resonance imaging in spinal cord injury without radiological abnormality in adults: a retrospective study. J Trauma Acute Care Surg 2013; 74: 845-848.

4 Boese CK, Lechler P. Spinal cord injury without radiologic abnormalities in adults: a systematic review. J Trauma Acute Care Surg 2013; 75: 320-330.

5 Machino M, Yukawa Y, Ito K, Nakashima H, Kanbara S, Morita D et al. Can magnetic resonance imaging reflect the prognosis in patients of cervical spinal cord injury without radiographic abnormality? Spine 2011; 36: E1568-E1572.

6 Boese CK, Oppermann J, Siewe J, Eysel P, Scheyerer MJ, Lechler P. Spinal cord injury without radiologic abnormality in children: a systematic review and meta-analysis. J Trauma Acute Care Surg 2015; 78: 874-882.

7 Kulkarni MV, Bondurant FJ, Rose SL, Narayana PA. 1.5 tesla magnetic resonance imaging of acute spinal trauma. Radiographics 1988; 8: 1059-1082.

8 Bozzo A, Marcoux J, Radhakrishna M, Pelletier J, Goulet B. The role of magnetic resonance imaging in the management of acute spinal cord injury. J Neurotrauma 2011; 28: 1401-1411.

9 Aebli N, Wicki AG, Ruegg TB, Petrou N, Eisenlohr H, Krebs J. The Torg-Pavlov ratio for the prediction of acute spinal cord injury after a minor trauma to the cervical spine. Spine J 2013; 13: 605-612.

10 Talbott JF, Whetstone WD, Readdy WJ, Ferguson AR, Bresnahan JC, Saigal R et al. The Brain and Spinal Injury Center score: a novel, simple, and reproducible method for assessing the severity of acute cervical spinal cord injury with axial T2-weighted MRI findings. J Neurosurg Spine 2015; 23: 495-504.

11 Mohanty SP, Bhat NS, Singh KA, Bhushan M. Cervical spinal cord injuries without radiographic evidence of trauma: a prospective study. Spinal Cord 2013; 51: 815-818.

12 Mahajan P, Jaffe DM, Olsen CS, Leonard JR, Nigrovic LE, Rogers AJ et al. Spinal cord injury without radiologic abnormality in children imaged with magnetic resonance imaging. J Trauma Acute Care Surg 2013; 75: 843-847.

13 Neva MH, Roeder CP, Felder U, Kiener B, Meier W, Perler M et al. Neurological outcome, working capacity and prognostic factors of patients with SCIWORA. Spinal Cord 2012; 50: 78-80.

14 Sharma S, Singh M, Wani IH, Sharma S, Sharma N, Singh D. Adult Spinal Cord Injury without Radiographic Abnormalities (SCIWORA): clinical and radiological correlations. J Clin Med Res 2009; 1: 165-172.

15 Liao CC, Lui TN, Chen LR, Chuang CC, Huang YC. Spinal cord injury without radiological abnormality in preschool-aged children: correlation of magnetic resonance imaging findings with neurological outcomes. J Neurosurg 2005; 103 (1 Suppl): 17-23.

16 Elrai S, Souei Mhiri M, Arifa Achour N, Mrad Daly K, Ben Hmida R, Jemni Gharbi H et al. MR imaging in spinal cord injury. J Radiol 2006; 87 (2 Pt 1): 121-126.

17 Quenum K, Coulibaly O, Arkha Y, Derraz S, El Ouahabi A, El Khamlichi A. Adult spinal cord injury with no radiographic bone lesion: analysis of nine consecutive patients. NeuroChirurgie 2011; 57: 46-50.

18 Sun LQ, Shen Y, Li YM. Quantitative magnetic resonance imaging analysis correlates with surgical outcome of cervical spinal cord injury without radiologic evidence of trauma. Spinal Cord 2014; 52: 541-546.

19 Como JJ, Samia H, Nemunaitis GA, Jain V, Anderson JS, Malangoni MA et al. The misapplication of the term spinal cord injury without radiographic abnormality (SCIWORA) in adults. J Trauma Acute Care Surg 2012; 73: 1261-1266.

20 Aebli N, Ruegg TB, Wicki AG, Petrou N, Krebs J. Predicting the risk and severity of acute spinal cord injury after a minor trauma to the cervical spine. Spine J 2013; 13: 597-604.

21 Chang V, Ellingson BM, Salamon N, Holly LT. The risk of acute spinal cord injury after minor trauma in patients with preexisting cervical stenosis. Neurosurgery 2015; 77. 561-565.

22 Ramon S, Dominguez R, Ramirez L, Paraira M, Olona M, Castello T et al. Clinical and magnetic resonance imaging correlation in acute spinal cord injury. Spinal Cord 1997; 35: 664-673.

23 Kwon SY, Shin JJ, Lee JH, Cho WH. Prognostic factors for surgical outcome in spinal cord injury associated with ossification of the posterior longitudinal ligament (OPLL). J Orthop Surg Res 2015; 10: 94.

24 Shen H, Tang Y, Huang L, Yang R, Wu Y, Wang P et al. Applications of diffusionweighted MRI in thoracic spinal cord injury without radiographic abnormality. Int Orthop 2007; 31: 375-383. 